Cinémas

Revue d'études cinématographiques

Journal of Film Studies

\title{
La mélancolie du nom. Cinéma et identité nationale
}

\section{Serge Cardinal}

Volume 8, numéro 1-2, automne 1997

Cinéma et mélancolie

URI : https://id.erudit.org/iderudit/024740ar

DOI : https://doi.org/10.7202/024740ar

Aller au sommaire du numéro

Éditeur(s)

Cinémas

ISSN

1181-6945 (imprimé)

1705-6500 (numérique)

Découvrir la revue

Citer cet article

Cardinal, S. (1997). La mélancolie du nom. Cinéma et identité nationale.

Cinémas, 8(1-2), 13-33. https://doi.org/10.7202/024740ar

\section{Résumé de l'article}

Dans cet article, l'auteur entreprend de décrire la mélancolie à l'oeuvre dans les relations entre le cinéma et l'identité nationale, et dans les discours théoriques sur ces relations. Cette entreprise l'amène à questionner les concepts de mentalité, de champ, de contexte, de réflexion, de réfraction, de pratique, etc., qui ont servi et servent encore à comprendre la formation d'une identité nationale à travers une cinematographie. 


\title{
La mélancolie du nom. Cinéma et identité nationale
}

\section{Serge Cardinal}

\begin{abstract}
RÉSUMÉ
Dans cet article, l'auteur entreprend de décrire la mélancolie à l'œuvre dans les relations entre le cinéma et l'identité nationale, et dans les discours théoriques sur ces relations. Cette entreprise l'amène à questionner les concepts de mentalité, de champ, de contexte, de réflexion, de réfraction, de pratique, etc., qui ont servi et servent encore à comprendre la formation d'une identité nationale à travers une cinématographie.
\end{abstract}

\section{ABSTRACT}

In this article, the author sets out to describe how melancholy enters into the relationships between cinema and national identity, as well as the theoretical discourses on these relationships. This undertaking leads him to interrogate the concepts of mentality, field, context, reflection, refraction, practice, etc., which have served and continue to serve our understanding of the formation of a national cinema through cinematography.

Ces pays, on le constatera, sont en somme parfaitement naturels. On les retrouvera partout bientôt... Naturels comme les plantes, les insectes, naturels comme la faim, l'habitude, l'âge, l'usage, les usages, la présence de l'inconnu tout près du connu. Derrière ce qui est, ce qui a failli être, ce qui tendait à être, menaçait d'être, et qui 
entre des millions de "possibles" commençait à être, mais n'a pu parfaire son installation...

Henri Michaux, Ailleurs

Selon Kant, et après lui Wittgenstein, le temps est malade du langage.

Daniel Fano, Un champion de mélancolie

Vous avez fini par placer le cinéma et l'identité nationale dans l'ombre projetée de ce qui fait défaut, de ce qui s'absente, de ce qui se perd, de ce qui est nié et entamé. Vous êtes arrivé à la conclusion que le propre du cinéma et de l'identité nationale est cette alternance subtile d'apparitions et de disparitions. À la question "Qu'est-ce que le cinéma?", vous n'avez su répondre qu'en évoquant le croisement de tous les autres arts. A la question "Qu'estce que l'identité nationale?", vous n'avez su décrire que l'hybridité, le cosmopolitisme et la mondialisation. Vous avez finalement désiré vous tenir à la limite d'une césure et d'un renversement entre présence et absence, identité et altérité, perception et projection. Vous avez finalement désiré saisir l'« image d'une nation " au moment où elle ne cesse de se déplacer, de s'échapper, de se diluer.

En fait, vous n'avez rien désiré; vous vous êtes soumis aux puissances d'un "piège à négatif». Le concept d'identité forme ce piège dans lequel s'engouffrent les différentes visées de votre projet de connaissance. Que vous essayiez de saisir l'identité nationale comme une mentalité réfléchie dans les films, comme des rapports sociaux réfractés dans une cinématographie, comme des pratiques singulières aboutissant à la construction de représentations collectives, vous avez chaque fois l'impression de laisser s'échapper la proie. C'est à croire que le concept même d'identité ne vous permettra jamais de comprendre et de décrire la nation que comme une entité en fuite.

Le concept d'identité ne vous semble tenir son efficacité que de ses multiples retournements et renversements. Lidentité affir- 
mée est aussitôt niée; à peine construites, ses limites et son unité se déplacent et se désarticulent. Si une identité est d'abord constituée dans une opposition avec ce qui n'est pas elle, le dehors glisse bientôt vers le dedans, transformant la polarité en dualité, la frontière en clivage, une différence avec l'étranger en une étrangeté à soi. Comment alors imaginer le cinéma et la nation épargnés par ce concept d'identité fondé sur le négatif? Vous ne pouvez plus affirmer candidement: "Voici le cinéma, voici la nation." Dans votre recherche d'une spécificité où le cinéma n'est ni tout à fait la photographie, ni tout à fait le théâtre, ni tout à fait le roman ni la peinture, mais un "texte introuvable" ou un "signifiant imaginaire", dans votre définition de la nation qui n'est ni simplement une communauté improbable, une société divisée, une culture plurielle, mais le cadre de toutes ces dispersions, vous avez déjà ressenti cette puissance d'attraction de l'entamé, du labile, de l'invisible.

Cette puissance du négatif, emportant avec elle l'identité, la nation et le cinéma, n'est pas non plus sans danger pour celui qui pense. Elle vous a entraîné, dans vos tentatives de définition des rapports entre le cinéma et l'identité nationale, à reconnaître la part d'ombre toujours déplacée de vos multiples constructions conceptuelles. L'histoire des mentalités, la théorie de l'action ou les études culturelles, les concepts de réflexion, de réfraction, de représentation collective, de champ, de contexte, etc., semblent vous reconduire toujours vers l'indicible, l'irreprésentable, l'insaisissable. Il vous semble que cette négativité généralisée vous condamne au bégaiement: votre discours est réduit au "ni... ni...", à la détermination négative qui ne cesse de poser une limite pour mieux la déplacer, qui ne cesse de lever l'endroit pour mieux révéler l'envers (le cinéma, ce n'est ni ceci ni cela; l'identité nationale, ce n'est ni ceci ni cela). Cette rhétorique est peut-être une rhétorique de l'échec, mais sans vouloir renoncer au savoir ni à l'analyse, vous ne voyez plus comment y échapper.

Ainsi, vous décidez de laisser le travail du négatif remettre en question une dernière fois les relations du cinéma à l'identité nationale, et les discours théoriques sur ces relations. Vous décidez de cerner à nouveau la logique du négatif à l'œuvre dans les 
productions imaginaires et critiques d'une société. Vous décidez de questionner les concepts de mentalité, de champ, de contexte, de réflexion, de réfraction, de pratique, etc., qui ont servi et servent encore à comprendre la formation d'une identité nationale à travers une cinématographie. C'est la capacité même de ces concepts à poser et à déplacer les limites d'une connaissance de l'identité qui vous invite à un réexamen.

Mais au moment de reprendre cette réflexion, vous soupçonnez secrètement sa vanité. Il faudrait reprendre ces questions beaucoup plus en amont, à leur foyer de détermination. Ce ne sont pas les réponses aux questions "Qu'est-ce que le cinéma?", "Qu'est-ce que l'identité nationale?" qui sont insuffisantes; c'est la question "Qu'est-ce que... ?" qui pose problème. Vous savez déjà que vous ne pourrez y répondre adéquatement. Cette question inévitablement vous pousse à l'incertitude, à l'impuissance, à la tristesse... à la mélancolie.

\section{L'attraction du vide}

Vous entreprenez d'abord de détisser les liens entre les films et une culture nationale pour en comprendre le tissu et la tessiture. Pour cela, vous parcourez un corpus filmique dans toute l'étendue de sa trame afin d'en dégager une constante qui expliquerait en entier et d'un seul coup une nation et ses films. Vous cherchez à révéler une dimension cachée: une mentalité.

Lidentité nationale est d'abord pour vous une conception du monde que possède une collectivité à un moment de son histoire. Une conception du monde qui se manifeste à travers les croyances, les mours, les productions artistiques, etc. C'est cette conception du monde que vous désignez sous le terme de mentalité. Vous postulez que la culture, et plus précisément les films, trouvent leur sens dans cette mentalité dont la première qualité est d'être latente. Aucun film n'exprime directement la mentalité, aucune analyse contemporaine d'un film non plus. Ils agissent à titre de traces ou d'échos d'une mentalité à interpréter ; vous avez à déchiffrer ces films et ces analyses pour révéler la mentalité. À votre avis, sous l'apparence affirmée de la culture, une mentalité servirait de plan originel accessible par le détour, le renvoi, le symptôme; sous les faits empiriques se tiendrait une 


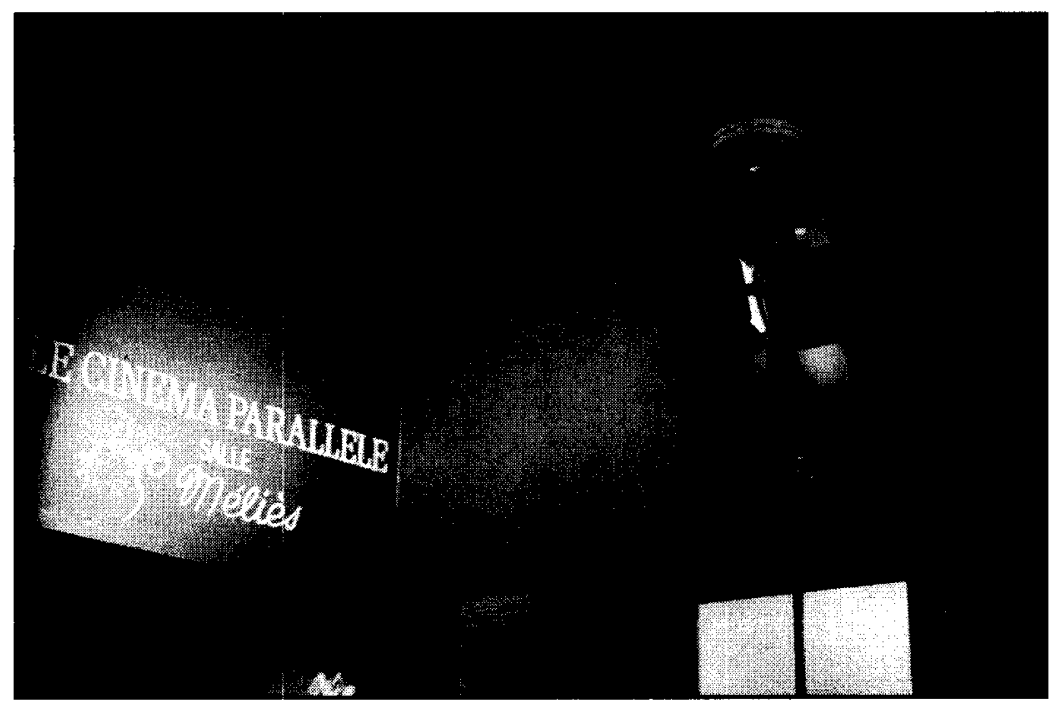

\section{Le cinéma Parallèle, Montréal}

Collection personnelle

sorte de «refoulement originaire» à circonscrire par le déchiffrement symptomal. Vous entreprenez de saisir une absence.

Votre entreprise n'a de chance de réussite que si elle rassemble le plus grand nombre de films (auxquels vous ajoutez bientôt d'autres artefacts) que vous considérez comme textes à déchiffrer, à comparer, à interpréter selon certaines règles permettant de cerner «[...] l'isomorphie profonde que vous supputez derrière la variété des pratiques [...]" (Duve, p. 11). Votre inventaire cherche à repérer des régularités qui ne seraient pas simplement le résultat d'une addition de tous les films que vous voyez, mais le dénominateur commun, le général qui se dégage de tous ces particuliers. Vous retrouvez la mentalité autant dans la production entière d'une époque ou d'un pays que dans l'œuvre complète d'un grand cinéaste ou du plus petit réalisateur de série $\mathrm{B}$.

$\mathrm{Au}$ passage se dressent quelques difficultés. Alors qu'il s'agit de tracer les limites d'une époque, vous ne savez plus où commence ni où finit une mentalité. Elle semble toujours trouver une assise plus loin en amont (dans la marginalité d'un groupe ou la singularité d'une œuvre) et se perpétuer sous une forme ou une autre en aval (ce que vous appelez un archaïsme ou un 
barbarisme). Alors qu'il s'agit de cerner son territoire, vous ne savez plus où commence ni où finit son influence. L'américanité, dans sa puissance d'expansion, vient infiltrer des mentalités plus aisément réductibles à des frontières géographiques strictes. Finalement, comme vous gardez encore un goût pour la créativité et la liberté d'un auteur, vous ne savez plus à quel point la mentalité subsume son œuvre.

Ces difficultés ne disqualifient pas le concept de mentalité (que vous admettez d'ailleurs définir avec grossièreté), mais fissurent déjà son unidimensionnalité d'une profondeur qui l'ouvre sur un dehors, des différences, des transformations. Non seulement êtes-vous disposé à admettre plusieurs mentalités, mais prenez-vous un malin plaisir à souligner des écarts et des glissements dans l'histoire. Vous ne renoncez pas cependant à la possibilité de rassembler des séries de caractéristiques et d'expliquer leur sens par une conception mentale qui les précède. Pour vous, les films ne manifestent pas encore la force de rapports sociaux, politiques et économiques, et ce dans la mesure où le social, le politique et l'économique sont eux-mêmes pris en écharpe par la mentalité. Qu'un organisme subventionnaire exige un certain type de film vous semble moins une influence directe et réelle que la preuve que toutes les sphères de la société sont structurées par une même conception du monde.

Vous choisissez avec soin l'ensemble de vos films. Vous y repérez des régularités thématiques (l'attente, l'exil ou l'errance), stylistiques (le baroque, le modernisme ou l'autobiographie) et formelles (la répétition, le plan-séquence interminable ou la dysnarration) qui manifestent un certain type de mentalité (mélancolique).

Ces régularités que vous extrayez de tous ces films doivent être distinctives, récurrentes et générales: vous cherchez ici à nommer une différence qui identifie tout un groupe sur toute une époque. Inévitablement, des difficultés de représentativité, d'exhaustivité et d'extensivité se lèvent. Au milieu d'un ensemble de films, dont vous avez peine à nommer les limites et à limiter le nombre, la détermination statistique et esthétique de régularités, pouvant éventuellement trouver écho dans l'ensemble de la culture nationale, devient vite un délire de spécificité. 
Des difficultés matérielles vous apparaissent subitement comme une aporie.

D'abord, vous ne savez plus décider à quel moment «[...] un ensemble de caractéristiques [peut] être considéré [suffisamment] récurrent et général pour être la preuve d'une mentalité sous-jacente distinctive [...]». Devrez-vous vous en tenir à votre simple jugement (Lloyd, p. 18) ? Est-ce encore votre jugement qui décidera si des différences thématiques, stylistiques et formelles renvoient à une seule mentalité ou si, au contraire, de fortes régularités renvoient à plusieurs mentalités?

Puis, vous remarquez que votre synthèse ne fait que rapporter à un niveau conceptuel supérieur (la mentalité) des régularités thématiques, stylistiques et formelles qu'une simple observation aurait pu répertorier. Vous pensiez découvrir des principes; vous n'avez fait qu'opérer une classification de différents phénomènes. Le déchiffrement, visant à cerner une mentalité postulée à travers ses reflets et ses traductions dans une série de films, touche ici à sa limite. Si la présupposition d'une mentalité permet le repérage de figures dominantes et récurrentes, cette " origine " présupposée n'explique en rien le fonctionnement de ces figures. Le rapprochement de ressemblances n'est que le début d'une histoire. Votre position théorique souffre d'un retournement tautologique. Vous ne pouvez inférer cette mentalité que des productions filmiques qui, de leur côté, ont besoin d'être expliquées par cette même mentalité pour acquérir quelque pertinence dans votre projet de connaissance. Vous cherchez à définir la mentalité qu'une société a manifestée à son insu et qui, à titre de secret, n'est accessible qu'à l'aide d'un déchiffrement. Vous n'avez d'autre choix que d'inférer cette mentalité de l'immobilité de la caméra, du thème de l'errance, du style baroque que vous envisagiez justement expliquer par cette mentalité (Panofsky, 1970).

Vous n'avez donc que des manifestations filmiques; vous cherchez encore ce qui se manifeste, car, jusqu'à présent, il n'y a qu'un vide où convergent toutes ces manifestations. La mentalité vous apparaît d'autant plus inexistante qu'elle fait signe partout, d'autant plus indécidable qu'elle peut tout définir. La mentalité est un écran vide qui attire à lui tous les films et autorise les rapprochements, les regroupements, les formations 
identitaires. Mais voulez-vous vraiment d'une nation qui se rassemble autour d'une absence?

\section{La fuite du lieu commun}

Vous décidez d'abandonner le concept de mentalité. L'identité nationale ne peut pas être cette conception du monde dont on arrive mal à déterminer la genèse, les limites, la structure exacte, la zone d'influence et la transformation. Vous ne pensez plus nécessaire de postuler "[...] une structure universelle, inconsciente et sous-jacente au corpus hétéroclite constitué [...]" que vous nommez un cinéma national (Duve, p. 11). Vous éviterez dorénavant tout recours à une condition transcendantale. Vous pensez plutôt qu'une identité nationale est à construire et qu'à ce projet, les films ne sont plus les surfaces de réflexion de l'"âme d'un peuple", mais les instruments d'un processus de construction à l'œuvre dans un espace social. Les films participent maintenant d'un rapport de forces entre plusieurs agents sociaux; ils deviennent des prises de positions conflictuelles prises par des membres d'une nation en perpétuelle transformation.

C'est dire à quel point vous ne vous contentez plus d'une relation biunivoque entre les films et l'identité nationale. Vous jugez la relation de représentation insuffisante (Récanati, 1979). Un film ne peut plus renvoyer de manière autonome à une identité. Il a besoin d'être expliqué, délimité, justifié. Tout est dans l'usage d'un film. L'identité nationale est une construction, l'aboutissement temporaire d'un processus de signification et de communication dont les films sont les instruments. Vous entendez tirer toutes les conséquences de cette nouvelle "prise de position" théorique.

Les films ne seront plus pour vous intrinsèquement représentatifs d'une identité nationale. La relation des films à l'identité sera médiatisée par l'usage intentionnel qu'en font les agents sociaux dans un certain contexte. Votre recherche sur les rapports entre cinéma et identité nationale ne tracera plus une correspondance entre des régularités formelles, thématiques et stylistiques et une mentalité ; elle analysera le point de vue à partir duquel un ensemble de films est perçu et utilisé dans la construction d'une identité. 
Mais alors que vous débutez votre description des rapports sociaux, des processus de signification et de communication qui mènent à la construction d'une identité nationale, vous soupçonnez déjà le moment où celle-ci ne se livrera plus dans toute la splendeur d'une affirmation, dans toute la réalité d'une manifestation identitaire explicite. Vous n'en avez pas fini avec le négatif. Plus vous vous penchez sur les pratiques de signification, plus l'identité perd de son évidence intuitive, de son immédiateté. Vous voyez combien d'interprétations et de contre-interprétations il faut pour produire une identité nationale à travers une pratique cinématographique, combien de constructions poétiques et d'artifices il faut pour former l'image d'une nation, à quel point cette image est liée à l'acte contingent dont elle est issue, à quel point elle est liée à des actions et à des réactions d'actants sociaux à un moment de l'histoire. C'est surtout la totale détermination de l'identité par la construction qui vous fait d'abord douter de son existence. Vous doutez plus précisément de ce qu'il faut entendre ici par existence. Qu'est-ce qu'une identité construite et contingente? Qu'est-ce qu'une identité comme pure création de celui qui s'identifie? Une hallucination? Vous regrettez un instant le concept de mentalité.

Si vous optez maintenant pour l'étude des processus de construction, pour l'étude de la production et de l'interprétation des images qu'une société effectue au moment de se définir, c'est que vous êtes dorénavant convaincu qu'une identité nationale n'existe pas en dehors des films (et plus largement, de toute production culturelle). Les films ne sont plus un ensemble à vocation figurative réfléchissant l'identité, mais des enjeux, des processus, des pratiques, des prises de positions à l'intérieur d'un contexte. L'identité n'est inscrite ni dans une réalité physique ou phénoménale, ni dans des représentations. Les objets que vous considérez utiles à la constitution d'une identité ne sont d'abord que des choses sans assignation avant de devenir des signes pour cette identité. Plus encore, lorsqu'une société se sert d'un ensemble de films pour signifier une identité, elle ne fait pas référence à un état de choses, mais à un objet de croyance. Et cette croyance ne pourra peut-être jamais se vérifier dans un état de faits; elle sera au mieux relayée et étayée par de nouvelles 
interprétations. Ne vous en déplaise, vous ne pourrez plus convoquer la réalité en contre-expertise. Vous êtes d'emblée dans l'imaginaire ou dans la "réalité " des constructions. Vous vous demandez si cela ne vous confine pas de nouveau dans un face à face avec l'absence, si le lien de ces constructions avec l'identité n'est pas l'équivalent de celui du mélancolique à l'objet perdu. Dans cette difficulté à nommer l'identité et la nation, le cinéma paraît entretenir une relation particulière avec la limite qui marque l'inaccessibilité, l'absence, le manque. Comme si, en dépit de son incertitude, le spectateur était condamné à maintenir le lien avec l'identité, à compenser le moment de la pure perte, par des constructions de plus en plus complexes, afin d'assurer un sens à la collectivité dans la mélancolie.

Vous avez sans doute mal évalué le virage dans lequel vous vous engagiez. Il n'est plus question ici d'identité, mais de jugement problématique: la proposition de simples possibilités à débattre. Ce qui rassemble des pratiques dans une nation ce n'est plus une essence, mais un débat commun. En témoignent les constructions, déconstructions, reconstructions nouvelles dont est l'objet ce que vous désigniez encore récemment comme l'identité nationale. La pluralité des actants et la multitude des processus de construction ne sont pas sans effet. A l'époque où vous preniez pour étalon de votre découpage la mesure géographique, vous aviez des chances d'aboutir à une unité sans doute fictive mais prévisible à votre perspective de départ. Vous pariiez qu'un rapprochement physique assurerait une communauté d'esprit. Si au contraire vous devenez attentif à l'histoire et à l'autonomie des champs (cinématographique, politique, économique) composant une société, si vous devenez attentif à leurs règles et à leurs enjeux propres, la possible rencontre de ces divers champs dans une problématique commune devient incertaine. "La question fondamentale devient alors de savoir si les effets de l'unité spatiale [...] sont assez puissants pour déterminer, par-delà l'autonomie des différents champs [...]" (Bourdieu, 1991, p. 18), non plus un esprit commun mais une problématique commune, un espace de possibles où puissent s'affronter des positions divergentes. Vous vous retrouvez dans cette position où, fier de votre approche des lieux de production 


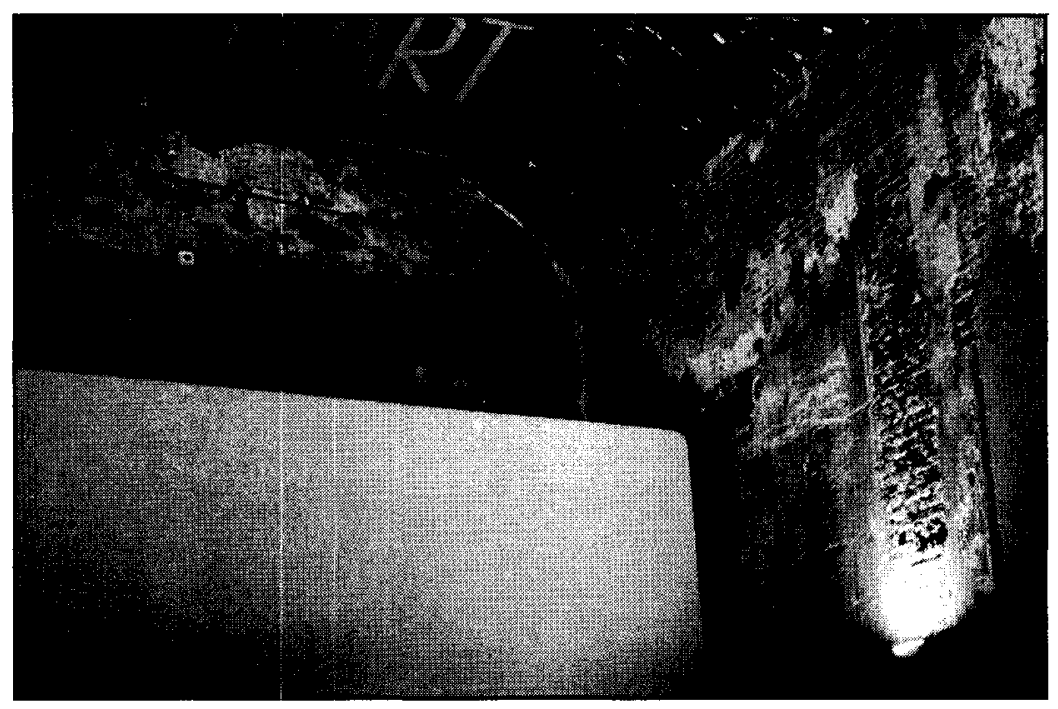

\section{Le cinéma Nova Bioskoop, Bruxelles}

Collection personnelle

de l'identité, vous perdez l'image de la nation. L'ensemble appelé "nation" vous apparaît comme une chimère ou comme une entité en sursis, un corps vide voué à la disparition dans l'affrontement des singularités. A moins qu'il ne s'agisse d'une union dans l'affrontement. Vous ne désespérez pas de cerner une communauté dans la manière qu'a une collectivité de s'affronter sur l'identité. La confiance en votre entreprise d'investigation des raisons pratiques vous permet d'échapper temporairement au doute hyperbolique: dans les constructions, déconstructions et reconstructions de l'identité se retrouve la force affirmative d'une production d'un "nous".

Vous commencez donc à opérer des comparaisons, non pas entre quelques traits isolés de la société et des films (vous évitez les équivalences entre une revendication politique et un trait stylistique), mais en comparant un ensemble de positions sociales à un ensemble de traits stylistiques, thématiques et formels, non plus considérés comme qualités esthétiques (vous n'avez pas l'ambition de créer un réseau intertextuel), mais comme prises de positions. Vous ne découvrez plus ainsi une sorte d'essence biologique ou culturelle, mais des structures, des mécanismes et 
des principes de construction de l'espace social commun. $\AA$ scruter ces comparaisons, vous devenez de plus en plus convaincu que l'espace social est premier et dernier : il détermine le sens que prendront les oppositions et les différences entre les styles, les thèmes et les formes d'une cinématographie.

Afin d'augmenter la précision de votre analyse, vous subdivisez l'espace d'une société en plusieurs champs. Le champ cinématographique a des enjeux communs avec l'ensemble de la société. Mais il a surtout ses visées propres qui lui permettront de devenir ce milieu de réfraction entre les films et la société. Les films n'exprimeront plus directement la société puisque entre eux se tient le champ cinématographique, qui recompose en partie selon ses besoins les enjeux traversant toute la société. La construction d'une identité devient de plus en plus le résultat de débats entre les agents cinématographiques se positionnant entre eux et par rapport aux agents d'autres champs englobants (social, politique, économique).

La récurrence du thème de l'errance ne prend alors son sens qu'en rapport à d'autres positions thématiques (le retour à la terre), politiques (la scission administrative du pays dans le marché mondial), sociales (l'ouverture à l'immigration dans la défense de la langue), économiques (la crise du secteur primaire et la montée des télécommunications), ethniques (l'épuration culturelle et la crise des réfugiés), géographiques (la transformation du territoire en lieu de transit ferroviaire), etc. La mélancolie n'apparaît plus comme une mentalité, mais comme une réponse possible à des changements dans les différentes sphères de la société. Les affects de perte, d'absence, de manque et d'abandon ne constituent plus les traits d'une identité essentiellement endeuillée, orpheline ou déficitaire, mais des réactions datées d'un groupe donné.

Comme vous ne reliez plus les films directement à une mentalité sous-jacente, vous ne reliez pas davantage les films aux caractéristiques sociales des auteurs, aux enjeux d'une classe, à l'idéologie d'un groupe. Les films sont les théâtres d'opérations d'un système d'écarts différentiels entre les multiples positions des agents de la société: 
Chaque prise de position (thématique, stylistique, etc.) se définir (objectivement et parfois intentionnellement) par rapport à l'univers des prises de positions [...] et par rapport à la problématique comme espace des possibles qui s'y trouvent indiqués ou suggérés; elle reçoit sa valeur distinctive de la relation négative qui l'unit aux prises de positions coexistantes [...] (Bourdieu, 1991, p. 19).

Sans raison apparente, cette complexité subitement vous attriste. Vous avez l'impression de perdre quelque chose que vous ne savez encore nommer. Si les films ne reflètent pas directement la société tout entière, ils ne peuvent pas non plus produire l'image d'une nation dans la mesure où ils sont déterminés par l'action des agents dans le champ cinématographique. Ils ne peuvent plus eux-mêmes ouvrir un espace de possibles puisqu'ils entrent dans un rapport d'homologie et de dépendance avec le champ cinématographique, là où se dessine réellement un système de coordonnées (les problèmes, les références, les repères, les concepts) définissant les directions possibles d'une construction identitaire :

Le moteur de changement des œuvres culturelles, langue, art, littérature, science, etc., réside dans les luttes dont les champs de production correspondants sont le lieu: ces luttes qui visent à conserver ou à transformer le rapport de forces institué dans le champ de production ont évidemment pour effet de conserver ou de transformer la structure du champ des formes qui sont des instruments et des enjeux dans ces luttes (Bourdieu, 1994, p. 91).

Ou vous avez mal compris, ou alors les films sont refoulés au rang d'instances secondes s'affichant sans opacité, sans diffraction, en toute transparence (Geertz, p. 99). Les films deviennent des illustrations de conceptions déjà à l'œuvre dans une microsociété. Si l'introduction des rapports sociaux vous arrache à la reproduction simple d'une mentalité monolithique, elle ne vous délivre pas du représentationnalisme. En vous débarrassant de la ressemblance, vous avez gardé l'analogie. Vous étiez précédemment laissé seul avec des manifestations filmiques sans référent, 
la mentalité toujours plus insaisissable dans l'absolu; vous perdez à présent le cinéma et vous retrouvez seul avec des luttes de pouvoir, de réputation, de distinction, de politique.

Vous vous demandez un instant si la peinture impressionniste, en dehors de ses batailles contre l'académisme et de son accrochage dans les salles de bains d'Amérique, a pu former, par son travail pictural sur la perception, une nouvelle vision du monde. Si le cinéma, en dehors de son combat entre esthétiques et son absorption par la télévision, a pu former, par son travail filmique sur l'identification, une identité nationale. Vous vous demandez si les films ne sont que des supports malléables d'une construction identitaire qui se joue ailleurs ou si, au contraire, cette logistique a son influence sur le symbolique, si la machinerie a une incidence sur la projection imaginaire d'un "nous" (Debray, p. 19). Non seulement la singularité des lieux et des communautés vous intéresse, mais aussi les formes dont usent les spectateurs dans la construction d'une identité. Sous prétexte d'analyser une illusion et de "[...] suspendre la relation de complicité et de connivence qui lie tout homme cultivé au jeu culturel $[\ldots]$ " (Bourdieu, 1991, p. 23), vous vous demandez si la réduction d'une cinématographie à un champ de bataille, où s'affrontent des agents sociaux dans la définition d'une identité nationale, ne vient pas trop diminuer la force des formes filmiques. Vous vous demandez si la prise en considération des longs plans-séquences, de l'errance et de la prolifération baroque (en dehors d'une histoire des formes) par d'autres productions culturelles, dans le cycle des interprétations (et non simplement des différenciations), n'a pas permis de construire une image de la communauté non réductible aux jeux des champs. Mais dénonçant ici le désenchantement de l'art, vous succombez à la mélancolie propre à votre champ, pour lequel toute perspective sociale de l'art équivaut à une perte d'aura. Vous n'acceptez pas de perdre le cinéma. Mais ce que vous appelez «la force des formes filmiques" (invention d'une perception, d'une affection et d'une conception spécifiques; formation singulière du temps et de l'espace) n'apparaît que dans la réfraction de votre champ. Elle n'a pas d'existence propre, ni aucune chance de perdurer dans votre champ en perpétuelle redéfinition. Cette "force" offre une 
possiblité bien incertaine à la projection de figures identitaires. Par ailleurs, pouvez-vous vraiment compter sur la formation, de film en film, de figures identitaires communes dans la mesure où chaque champ de la société opère selon ses propres paramètres? Il ne vous reste plus qu'à comprendre comment chaque microsociété se forme sa propre image d'une nation sans identité.

Toute la question est alors de savoir où tracer la limite de chaque contexte singulier et quels éléments y inclure. Si la construction d'une irnage identitaire est un "[...] processus historiquement déterminé dont les modes et les modèles varient selon les temps, les lieux, les communautés" (Chartier, p. 1509), encore faut-il pouvoir le saisir et l'expliquer.
A défaut d'être spécifiée adéquatement, la notion de contexte risque de ne rien faire d'autre que de répéter inlassablement un truisme aux multiples figures, qui dirait que la production des énoncés est soumise à un certain nombre de contraintes et de déterminations, qu'elle ne survient pas dans des circonstances indiffé- rentes, que le sens des énoncés dépend des conditions dans lesquelles ils apparaissent, etc." (Latraverse, p. 194-195).

Mais vous butez très tôt contre d'autres difficultés. Vous notez d'abord la multiplicité des éléments pouvant jouer un rôle actif dans le processus de signification circonscrit dans le temps et l'espace. C'est à croire que tout ce que vous identifiez à l'intérieur d'une situation de communication peut figurer comme l'un de ces éléments (Latraverse, p. 197). Le contexte se creuse à l'infini : des conditions spatio-temporelles, aux intentions poursuivies, affichées ou pressenties, aux connaissances, aux croyances, au rôle des partenaires en jeu dans l'interprétation, à ce qui détermine leur relations, à ce qui a précédé ou est contemporain, au paratexte, au cotexte, etc., la réception d'une série de films à une époque et un lieu donnés se pluralise, se complexifie. D'autant plus que ces éléments (et la liste n'est pas exhaustive), en plus d'interagir, "[...] se composent en fait d'un ensemble ouvert d'autres éléments au nombre difficilement spécifiable et à l'identité plus ou moins descriptible» (Latraverse, p. 197). 
Au moment où vous voulez approfondir la construction spécifique d'un groupe, vous plongez dans un gouffre sans fond. Non seulement avez-vous du mal à déterminer les éléments actifs dans la production de sens, mais vous ne voyez plus où tracer la limite du contexte. Après avoir perdu la pérennité de l'identité nationale dans l'invisibilité de la mentalité, sa totalité dans l'affrontement des champs, voilà que vous n'arrivez plus à saisir les étapes du mouvement menant à la construction d'identités singulières que vous auriez pu rapprocher pour produire le paysage différencié d'un cadre géopolitique appelé "la nation".

Vous songez alors que, comme tout le reste, un contexte pertinent peut se dégager de l'infini. Une hiérarchie des éléments est sans doute possible afin de dégager ceux qui agissent réellement dans la construction d'une image de la nation par un groupe donné. Avec l'ambition de faire ressortir la diversité et de mieux saisir le mouvement des interprétations, vous pratiquez ce qu'un chercheur appelle un "terrorisme de la petite dimension" (Reichler, 1995). Après la réflexion et la réfraction, vous passez à la raréfaction. Vous cherchez à vous rapprocher au plus près d'un contexte de base qui se confondrait avec la réalité d'une microsociété souvent réduite à une ethnie, à une classe, à un sexe, à un groupe test en vase clos durant une période très brève et interprétant à partir d'un minimum de textes filmiques.

Mais la diversité des résultats devrait vous rappeler qu'il n'y a pas de contexte naturel, qu'il n'y a que des "[...] opérations, des procédures, des expériences de contextualisation [...]" (Lahire, p. 393) produisant des effets de connaissance. Si une communauté construit une identité, c'est que vous avez d'abord construit cette communauté. Il n'y a pas de niveau d'analyse ou "[...] une échelle du contexte qui permettrait d'accéder aux pratiques réelles, aux pratiques “telles qu'elles sont”"(p. 398). Vous pensiez toucher l'identité réelle du doigt; vous étiez dans un délire hallucinatoire. Encore une fois, vous avez perdu.

Vous vous rendez compte alors que votre choix d'objet n'était pas indifférent à votre quête et à sa manière. Dès lors que vous posez la question de l'identité (nationale) en suivant le cours d'une pensée qui a besoin de la négation pour instituer une entité, le péril est réel: vous vous trouvez en position de subir 
un renversement: dialectique menant jusqu'à la disparition; vous aboutissez nécessairement à la perte, à l'absence, au manque, à l'abandon. Votre entreprise de saisissement est vouée à la faillite.

Avouez-le, vous étiez dès le départ cet éternel «[...] inconsolable qui se console, qui se prend par la main pour aller au travail", vous saviez secrètement que les "[...] objets perdus sont les seuls que l'on ait peur de perdre [...]" (Metz, p. 109).

\section{La mélancolie du non}

Dans votre projet de connaisance, l'identité nationale est soutenue par deux "fausses" affirmations: la représentation et la pratique signifiante. Telles que vous les définissez, la représentation devient invérifiable et la pratique, insaisissable. Cette véracité et cette positivité déchues sont les produits du négatif inaugural.

Plus vous avez essayé de saisir la représentation de l'identité nationale dans un corpus filmique, plus vous l'avez perdue comme réalité positive. Et lorsque vous avez tenté de ressaisir cette réalité dans la pratique signifiante, vous n'avez trouvé que du transitoire. De film en film, l'identité nationale est apparue comme une entité plus ou moins réalisée, toujours sur le point d'apparaître définitivement sans jamais y parvenir totalement. À croire que le signe d'une identité nationale ne peut être que travaillé par le négatif, et cela dès son origine.

Sans doute pensez-vous " [...] qu'il n'y a de sens que du désespoir", sans doute pensez-vous que seuls "[...] la perte, le deuil, l'absence [peuvent déclencher] l'acte imaginaire et le [nourrir] en permanence [...]", qu'il faut "[...] la nécessité du manque pour que surgisse le signe [...]" (Kristeva, p. 15 et 35), que le processus de signification est l'équivalent d'une dénégation de la perte, une manière de "retrouver» dans les signes une réalité toujours déjà perdue.

Mais cette inauguration place la signification au-dessus d'un gouffre. Elle court toujours le risque de s'abîmer dans cette absence. La signification est une lutte continuelle contre l'«effondrement symbolique» (p. 35), contre ce moment où la capacité de compensation du signe, où sa relation à l'objet sera mise en doute, laissant alors à découvert la perte. Si le symbole 
"[...] se constitue de dénier la perte [...]" (p. 38), le désaveu du symbole lui-même vous entraîne dans un deuil impossible. A l'origine de la signification se trouve la négativité, dans son effondrement, la mélancolie. C'est vers cet effondrement que vous vous êtes aventuré comme vers son destin un condamné, voire un fautif.

Alors que vous tentiez de saisir l'identité nationale à travers un ensemble de films, vous n'avez trouvé que fragments auxquels manquait la totalité, vous n'avez trouvé que mouvements interprétatifs auxquels manquait une direction. Au risque de verser dans le néant, vous avez tenté de resserrer votre acte d'appropriation ou de réappropriation autour d'un objet de plus en plus ténu. Chaque fois, vous vous êtes retrouvé avec une identité indéfinie, un signe en suspension qui détermine dans le présent une instabilité et dans le passé une incertitude, qui fonctionne comme une demande de sens, mais qui repousse toute assignation.

Mais pourquoi exigiez-vous de ramener la multiplicité à la division, la différence à l'opposition? Mais pourquoi interprétiez-vous le devenir et la transformation comme une dépossession dans le temps?

Sans doute est-il insuffisant de multiplier des différences, tâchant de ressaisir chacune d'elles dans sa limite, son unité, son identité, construisant de la sorte une vaste structure de distribution des productions identitaires, reportant la stabilité d'une identité sur des unités de production de plus en plus petites à mesure que l'ensemble devient de plus en plus incertain. Sans doute ne suffit-il pas de multiplier les catégories puisque chaque nouvelle division repose la question d'un Tout, chacune à son échelle. Surtout, il ne suffit pas de comprendre les différences comme une série d'oppositions. Sortir du non.

Il faudrait pouvoir considérer des particularités comme des singularités qui ne souffrent pas d'une totalité qui leur manque, qui leur aurait toujours manqué. Échanger le multiple contre la multiplicité, et retrouver ainsi ce qui, dans l'efficacité du concept de mentalité, attirait le rapprochement du "dispars». Mais un rapprochement qui, justement, n'élimine plus la différence, un rapprochement qui, au contraire, n'est possible que dans et 
par la différence, créant un "rhizome" où les productions identitaires sont dissymétriques, pièces de puzzle qui ne viennent pas du Même, mais de différents puzzles, violemment insérées les unes dans les autres, toujours locales mais jamais spécifiques, et leurs bords discordants toujours forcés, imbriqués les uns dans les autres, ou alors dans des rapports de contiguïté qui marquent des distances, dans des rapports d'éloignement qui produisent des connexions. Faire d'une totalité, une production, le résultat des pratiques signifiantes : faire de l'identité, un résidu. Sortir du nom.

\begin{abstract}
Nous ne croyons plus en ces faux fragments qui, tels les morceaux de la statue antique, attendent d'être complétés et recollés pour composer une unité qui est aussi bien l'unité d'origine. Nous ne croyons plus à une totalité originelle ni à une totalité de destination. Nous ne croyons plus à la grisaille d'une fade dialectique évolutive, qui prétend pacifier les morceaux parce qu'elle en arrondit les bords. Nous ne croyons à des totalités qu'à côté. Et si. nous rencontrons une telle totalité à côté de parties, c'est un tout de ces parties-là, mais qui ne les totalise pas, une unité de toutes ces parties-là, mais qui ne les unifie pas, et qui s'ajoute à elles comme une nouvelle partie composée à part (Deleuze et Guattari, p. 50-51).
\end{abstract}

Université du Québec à Montréal

\title{
BIBLIOGRAPHIE
}

Ang, Ien. "Hegemony-in-Trouble. Nostalgia and the Ideology of the Impossible in European Cinema ", dans Duncan Petrie (direction), Screening Europe. Image and Identity in Contemporary European Cinema. London: BFI Publishing (1992), p. 21-31.

Bellemare, Denis. "La perception projetée". Protée, vol. 23, n* 1 (1995), p. 45-50.

Bennington, Geoffrey. "Postal Politics and the Institution of the Nation ", dans Homi K. Bhabha (direction), Nation and Narration. London: Routledge (1990), p. 121 137.

Bhabha, Homi K. "DissemiNation: Time, Narrative, and the Margins of the Modern Nation ", dans Homi K. Bhabha (direction), Nation and Narration. London: Routledge (1990), p. 291-322.

Bourdieu, Pierre. Raisons pratiques. Sur la théorie de l'action. Paris: Seuil, 1994.

Bourdieu, Pierre. "Le champ littéraire". Actes de la recherche en sciences sociales, $\mathrm{n}^{\text {" }} 89$ (1991), p. 3-46. 
Boureau, Alain. «Propositions pour une histoire restreinte des mentalités". Annales, n" 6 (1989), p. 1491-1504.

Brennan, Timothy. "The National Longing for Form», dans Homi K. Bhabha (direction), Nation and Narration. London: Routledge, 1990, p. 44-70.

Caughie, John. "Becoming European. Art Cinema, Irony and Identity", dans Duncan Petrie (direction), Screening Europe. Image and Identity in Contemporary European Cinema. London: BFI Publishing (1992), p. 32-44.

Chambers, Ross. Mélancolie et opposition. Les débuts du modernisme en France. Paris: José Corti, 1987.

Chartier, Roger. "Le monde comme représentation». Annales, n' 6 (1989), p. 15051520.

Chouvier, Bernard. "Le monde en négatif ou les géographies imaginaires: de quelques inventions de pays et de peuples chez Beckett, Borges et Michaux ", Pouvoirs du négatif dans la psychanalyse et la culture (collectif). Seyssel: Champ Vallon (1988), p. $177-194$.

Debray, Régis. "La statue descellée pas ses socles même". Les Cabiers de médiologie, n"3 (1997), p. 17-27.

Deleuze, Gilles et Guattari, Félix. L'Anti-CEdipe. Capitalisme et schizophrénie. Paris: Minuit, 1972.

Duve, Thierry de. Au nom de l'art. Pour une archéologie de la modernité. Paris: Minuit, 1989.

Fano, Daniel. Un champion de mélancolie. Le Muy : Éditions Unes, 1985.

Fédida, Pierre. L'Absence. Paris: Gallimard, 1978.

Freud, Sigmund. "Deuil et mélancolie (1917)", CEuvres complètes, tome XII. Paris: P.U.F. (1988), p. 259-278.

Frodon, Jean-Michel. "La projection nationale. Cinéma et nation". Les Cahiers de médiologie, n"3 (1997), p. 135-145.

Geertz, Clifford. "Art as a Cultural System", Local Knowledge. Further Essays in Interpretative Antrhopology. New York: Basic Books (1983), p. 94-120.

Green, André. Le Travail du négatif. Paris: Minuit, 1993.

Gros, Frédéric. "L'effondrement comme fondement: pour une figure de la négation pure». Textuel, n²9 (1995), p. 151-156.

Hunt, Lynn. "Introduction: History, Culture, and Text", dans Iynn Hunt (direction), The New Cultural History. Berkeley: University of California Press (1989), p. 1-22.

Kristeva, Julia. Saleil noir. Dépression et mélancolie. Paris: Gallimard, 1987.

Lagny, Michèle. De l'Histoire du cinéma. Méthode historique et histoire du cinéma. Paris: Armand Colin, 1992.

Lahire, Bernard. "La variation des contextes en sciences sociales. Remarques épistémologiques". Annales, n² (1996), p. 381-407.

Laplanche, J. et Pontalis, J.-B. Vocabulaire de la psychanalyse. Paris: P.U.F., 1981.

Latraverse, François. La Pragmatique. Histoire et critique. Bruxelles: Pierre Mardaga, 1987.

Le Goff, Jacques. "Les mentalités. Une histoire ambiguë", dans Jacques Le Goff et Pierre Nora (direction), Faire de l'histoire, tome 3. Nouveaux objets. Paris: Gallimard (1974), p. 76-94.

Lloyd, Geoffrey E.R. Pour en finir avec les mentalités. Paris: La Découverte, 1996. Maupertuis. "Réflexions philosophiques sur l'origine des langues et la signification des mots (1748) ", Sur l'origine du langage. Genève: Librairie Droz (1971), p. 27-57. 
Metz, Christian. Le Signifiant imaginaire. Psychanalyse et cinéma. Paris: Bourgois, 1993.

Michaux, Henri. Ailleurs. Paris: Gallimard, 1967.

Panofsky, Erwin. "Le concept de Kunstwollen", La Perspective comme forme symbolique et autres essais. Paris: Minuit (1975), p. 197-221.

Panofsky, Erwin. Architecture gothique et pensée scolastique. Paris: Minuit, 1970.

Petrie, Duncan. "Introduction: Change and Cinematic Representation in Modern Europe", dans Duncan Petrie (direction), Screening Europe. Image and Identity in Contemporary European Cinema. London: BFI Publishing (1992), p. 1-8.

Récanati, François. La Transparence et l'énonciation. Pour introduire à la pragmatique. Paris: Seuil, 1979.

Reichler, Claude. "Introduction. Représentation et médiation symbolique dans la littérature de voyage ". Énudes de lettres (janv.-fév. 1995), p. 1-12.

Revel, Jacques. "Mentalités", dans André Burguière (direction), Dictionnaire des sciences historiques. Paris: PUF (1986), p. 450-456.

Rosen, Philip. "History, Textuality, Nation: Kracauer, Burch, and Some Problems in the Study of National Cinema ". IRIS, vol. 2, n”2 (1984), p. 69-84.

Rosenberg, Benno. "Pulsion de mort, négation et travail psychique: ou la pulsion de mort mise au service de la défense contre la pulsion de mort", Pouvoirs du négatif dans la psychanalyse et la culiure (collectif). Seyssel: Champ Vallon (1988), p. 65-73.

Rosenberg, Benno. "Le travail de mélancolie ou la fonction élaborative de l'identification ou le rôle du masochisme dans la résolution de l'accès mélancolique». Revue française de psychanalysi, $\mathrm{n}^{\circ} \mathrm{6}$ (1986), p. 1521-1543.

Rosolato, Guy. «Le négatif et son lexique», Le Négatif. Figures et modalités (collectif). Paris: Dunod (1989), p. 9-22.

Rosolato, Guy. "La psychanalyse du négatif». Topique, n" 18 (1977), p. 11-29.

Sojcher, Frédéric (direction). "Cinéma européen et identité culturelle". Revue de l'Université de Bruxelles, $\mathrm{n}^{\circ \mathrm{s}}$ 1-4 (1995), p. 5-16.

Sorlin, Pierre. Italian National Cinema, 1896-1996. London-New York: Routledge, 1996.

Sorlin, Pierre. European Cinemas, European Societies, 1939-1990. London: Routledge, 1991.

Sorlin, Pierre. Sociologie du cinéma. Onverture pour l'histoire de demain. Paris : Aubier, 1977.

Starobinski, Jean. "Lirnmortalité mélancolique». Le Temps de la réflexion, n" 3 (1982), p. 231-251. 